\title{
Pulmonary metastases in differentiated thyroid cancer: efficacy of radioiodine therapy and prognostic factors
}

\author{
Hong-Jun Song, Zhong-Ling Qiu, Chen-Tian Shen, Wei-Jun Wei and Quan-Yong Luo \\ Department of Nuclear Medicine, Shanghai Sixth People's Hospital, Shanghai Jiao Tong University, 600 Yishan Road, \\ Shanghai 200233, People's Republic of China
}

Correspondence should be addressed to Q-Y Luo

Email

lqyn@sh163.net

\begin{abstract}
Context: Data from a large cohort of patients with pulmonary metastases from differentiated thyroid cancer (DTC) were retrospectively analyzed.

Objective: To assess the effect of radioiodine therapy and investigate the prognostic factors of survival for patients with pulmonary metastasis secondary to DTC.

Methods: A total of 372 patients with pulmonary metastasis from DTC treated with ${ }^{131}$ I entered the study. According to the results of ${ }^{131}$ I whole-body scan (WBS), pulmonary metastases were classified as ${ }^{131}$ I-avid and non- ${ }^{131}$ I-avid. For patients with ${ }^{131}$ I-avid lung metastases, treatment response was measured by three parameters: serum thyroglobulin (Tg) levels, chest computed tomography (CT) and post-therapeutic ${ }^{131} \mathrm{I}$-WBS. Overall survival was calculated by the Kaplan-Meier method. Factors predictive of the outcome were determined by multivariate analyses.

Results: Among patients demonstrating ${ }^{131}$ I-avid pulmonary metastases (256/372, 68.8\%), 156 cases $(156 / 256,60.9 \%)$ showed a significant decrease in serum Tg levels after ${ }^{131}$ I therapy and 138 cases $(138 / 229,60.3 \%)$ showed a reduction in pulmonary metastases on follow-up CT. A complete cure, however, was only achieved in 62 cases $(62 / 256,24.2 \%)$. Multivariate analysis showed that only age, the presence of multiple distant metastases and pulmonary metastatic node size were significant independent variables between the groups of ${ }^{131} \mathrm{I}$-avid and non- ${ }^{131} \mathrm{I}$-avid.

Conclusion: This study indicated that, most ${ }^{131} \mathrm{I}$-avid pulmonary metastases from DTC can obtain partial or complete remission after ${ }^{131}$ I therapy. Younger patients ( $<40$ years old) with only pulmonary metastases and small ('fine miliaric' or micronodular) metastases appear to have relative favorite outcomes. Patients who do not respond to ${ }^{131}$ I treatment have a worse prognosis.
\end{abstract}

European Journal of

Endocrinology

(2015) 173, 399-408

\section{Introduction}

Differentiated thyroid cancer (DTC) is most commonly confined to the thyroid gland with or without involvement of regional lymph nodes because of its typical slowgrowing pattern. Distant metastases are an uncommon event in DTC and a wide range in prevalence (4-23\%) has been reported $(1,2,3,4,5,6)$. Lungs are the most frequent site of distant metastasis in DTC and the resulting respiratory failure may be the most common cause of death. About $50 \%$ of patients with such metastases die within 10 years (7). Postoperative ablation therapy with
${ }^{131} \mathrm{I}$ is a mainstay of treatment in such cases, in conjunction with complete surgical resection of the thyroid and thyrotropin (TSH) suppression with thyroxine $\left(\mathrm{T}_{4}\right)$. According to iodine-concentrating capacity (8), pulmonary metastases are classified as ${ }^{131}$ I-avid and non- ${ }^{131}$ I-avid. ${ }^{131} \mathrm{I}$ therapy is only recommended for patients with ${ }^{131} \mathrm{I}$-avid lung metastases, and there is no obvious benefit for those with non- ${ }^{131}$ I-avid metastases. Thus, the characteristics and prognosis differ between the two groups. However, few previous investigations have

Published by Bioscientifica Ltd. 
discussed the efficacy of radioiodine therapy and prognostic factors of ${ }^{131} \mathrm{I}$-avid and non- ${ }^{131} \mathrm{I}$-avid lung metastases separately.

Serum thyroglobulin (Tg) level is a useful prognostic factor after radioactive iodine ablation and its measurement is included in the follow-up DTC patients according to the guidelines of the British Thyroid Association and the American Thyroid Association (9, 10). Conventional chest X-ray is routinely used for diagnosis and follow-up of DTC patients with pulmonary metastases. However, pulmonary metastases are more often encountered in patients with papillary carcinoma and their radiological size is usually $<1 \mathrm{~cm}$ and frequently below the spatial resolution of the chest X-ray. Chest computed tomography (CT) is preferred over chest X-rays and it enhances the diagnostic accuracy in most patients with normal chest X-ray findings (11). Thus, it is useful for the detection of pulmonary metastases of DTC and is able to measure the node size of metastases accurately.

In this study, data from a large cohort of patients with pulmonary metastasis from DTC treated and managed at the same institution were retrospectively analyzed. We aimed to: i) asses the therapeutic effects of radioiodine treatment on ${ }^{131} \mathrm{I}$-avid pulmonary metastases by the change of Tg level and node size; ii) investigate the prognostic factors related to ${ }^{131} \mathrm{I}$-avid pulmonary metastases; iii) compare the significant factors that affect the ability of ${ }^{131}$ I uptake between patients with ${ }^{131}$ I-avid and non- ${ }^{131}$ I-avid pulmonary metastases and iv) evaluate the overall survival (OS) and the variables influencing survival.

\section{Subjects and methods}

\section{Patients}

From January 1997 to December 2013, 4681 patients with DTC were treated at our institution and 372 (372/4681, $7.95 \%$ ) who had lung metastases yielded the final cohort with the average age of $54.25 \pm 16.08$ years (range, $12-86$ years). Primary thyroid cancers were papillary in 288 patients and follicular in 84 . Only pulmonary metastases were involved in 298 patients while 74 patients also had distant metastases to other organs.

\section{Diagnostic criteria for DTC pulmonary metastases}

Definitive cytological or pathologic confirmation was not possible in all patients; therefore, diagnosis of pulmonary metastases was based on clinical and postoperative or follow-up chest CT findings, diagnostic or therapeutic ${ }^{131} \mathrm{I}$ whole-body scan (WBS) and serum Tg levels. A patient satisfying the following criteria was considered to have pulmonary metastases: i) pathological results confirmed; ii) pulmonary ${ }^{131} \mathrm{I}$ uptake on ${ }^{131} \mathrm{I}-\mathrm{WBS}+$ pulmonary node(s) on chest CT+elevated serum Tg level; iii) pulmonary ${ }^{131} \mathrm{I}$ uptake on ${ }^{131} \mathrm{I}-\mathrm{WBS}+$ normal chest $\mathrm{CT}+$ elevated serum $\mathrm{Tg}$ level or iv) no pulmonary ${ }^{131} \mathrm{I}$ uptake on ${ }^{131} \mathrm{I}$-WBS + pulmonary nodes on chest $\mathrm{CT}+$ elevated serum Tg level.

According to the results of ${ }^{131} \mathrm{I}-\mathrm{WBS}$, pulmonary metastases were classified as ${ }^{131}$ I-avid (functioning pulmonary metastases) and non- ${ }^{131}$ I-avid (non-functioning pulmonary metastases). According to the chest CT results, patients were divided into three categories: i) included patients with negative chest CT but ${ }^{131}$ I uptake on ${ }^{131}$ I-WBS, defined as 'fine miliaric'; ii) included patients with micronodular pulmonary metastases, defined radiologically as $\leq 1 \mathrm{~cm}$ in diameter and iii) included patients who had macronodular pulmonary metastases, defined radiologically as $>1 \mathrm{~cm}$ in diameter. According to the discovery time, pulmonary metastases were defined as initial (found at the time of or within 6 months after thyroidectomy) and developed (found 6 months or more after thyroidectomy).

\section{Procedures for radioiodine therapy}

Two hundred and seven (55.65\%) patients received unilateral total and contralateral subtotal thyroidectomy and total thyroidectomy was performed in 165 (44.35\%) cases. The patients achieved a sufficient hypothyroid state clinically with a serum TSH level $>30 \mu \mathrm{IU} / \mathrm{ml}$. All patients were instructed to follow a low-iodine diet for at least 2 weeks. The dose of ${ }^{131}$ I was 3.7-7.4 GBq (100-200 mCi). Conventional measurements, including free tri-iodothyronine, free $\mathrm{T}_{4}, \mathrm{TSH}, \mathrm{Tg}, \mathrm{Tg}$ antibody (TgAb), neck ultrasonography and chest CT scan were performed before ${ }^{131}$ I administration. Tg and TgAb were measured by RIA (from 1997 to 1999) and chemiluminescence (from 1999 to 2013). All patients underwent conventional CT for examination of metastatic lesions without the use of contrast media in order not to affect the radioiodine therapy. The CT images were obtained with $7 \mathrm{~mm}$ slice thickness starting from the apex of the lungs. All CT images were obtained with the patient in the supine position. Patients were evaluated in terms of metastatic pulmonary lesions and the lesions were classified according to size. Levothyroxine $\left(\mathrm{L}-\mathrm{T}_{4}\right)$ therapy was replaced $48 \mathrm{~h}$ later and post-therapeutic ${ }^{131} \mathrm{I}$-WBS was carried out 5 days after ${ }^{131}$ I administration. Post-treatment ${ }^{131} \mathrm{I}$-WBS was obtained in the anterior and posterior projections 
with a large field-of-view gamma camera equipped with a high-energy (peak energy centred on $360 \mathrm{keV}$ with a $20 \%$ energy window), parallel-hole collimator (Hawkeye Millennium VG, GE Medical Systems, Milwaukee, WI, USA). In patients with ${ }^{131} \mathrm{I}$-avid metastases on ${ }^{131} \mathrm{I}$-WBS, additional treatments with ${ }^{131}$ I were given $4-12$ months later. In cases with considerable residual thyroid tissue, even post-therapeutic WBS may fail to show ${ }^{131}$ I concentration in the lungs owing to significant neck uptake (12), therefore, ${ }^{131}$ I therapy was discontinued in patients with non- ${ }^{131}$ I-avid lung metastases after residual thyroid tissue was completely ablated.

\section{Evaluation of changes in serum Tg level}

After at least two courses of ${ }^{131}$ I therapy, changes in Tg level were classified into three types: effectiveness (biochemical relief (BR)), stabilization and ineffectiveness according to our previously study (13). i) Effectiveness: compared with pretreatment, the serum Tg levels exhibited a reduction of $\geq 25 \%$; ii) stabilization: compared with pretreatment, the serum Tg decreased or increased by $<25 \%$ and iii) ineffectiveness: compared with pretreatment, the serum $\mathrm{Tg}$ increased by $\geq 25 \%$.

\section{Evaluation of changes in chest CT images}

Chest CT responses were assessed using Response Evaluation Criteria in Solid Tumors (RECIST, version 1.1) as follows: i) complete response (CR), disappearance of all lesions; ii) partial response (PR), $\geq 30 \%$ decrease in the sum of lesions diameters, taking as reference the baseline sum diameters; iii) progressive disease (PD), $\geq 20 \%$ increase in the sum of lesion diameters or appearance of greater than or equal to one new lesions is also considered progression and iv) stable disease (SD), neither sufficient shrinkage to qualify for PR nor sufficient increase to qualify for PD.

\section{Criteria for complete cure}

Complete cure was defined by all the following criteria: no evidence or minimal visual evidence of ${ }^{131}$ I concentration on ${ }^{131} \mathrm{I}$-WBS in the chest region; no evidence of residual disease

Table 1 Changes of serum Tg for 256 DTC patients with ${ }^{131} \mathrm{I}$-avid lung metastases after ${ }^{131} \mathrm{I}$ therapy: univariate statistical analysis.

\begin{tabular}{l}
\hline Factors \\
\hline Age $^{\mathrm{a}}$ \\
$0-19$ \\
$20-39$ \\
$40-59$ \\
$\geq 60$ \\
Gender \\
Male \\
Female \\
Pathological type \\
Papillary \\
Follicular \\
Operation method \\
Lobectomy or subtotal thyroidectomy \\
Total thyroidectomy \\
Time of lung metastasis \\
Initial \\
Developed \\
Tg level \\
$<100$ \\
100-1000 \\
$>1000$ \\
Metastatic site \\
Lung and lung + lymph node \\
Lung + bone and lung + other organs \\
CT results \\
Positive \\
Negative \\
Total
\end{tabular}

\begin{tabular}{c} 
No. of patients \\
\hline $18(7.03 \%)$ \\
$112(43.75 \%)$ \\
$86(33.59 \%)$ \\
$40(15.63)$ \\
$95(37.11 \%)$ \\
$161(62.89 \%)$ \\
$193(75.39 \%)$ \\
$63(24.61 \%)$ \\
$140(54.69 \%)$ \\
$116(45.31 \%)$ \\
$39(15.23 \%)$ \\
$217(84.77 \%)$ \\
$41(16.02 \%)$ \\
$141(55.08 \%)$ \\
$74(28.90 \%)$ \\
$197(76.95 \%)$ \\
$59(23.05 \%)$ \\
$229(89.45 \%)$ \\
$27(10.55 \%)$ \\
256 \\
\hline
\end{tabular}

\begin{tabular}{c}
\hline Effective \\
\hline 10 \\
71 \\
57 \\
18 \\
65 \\
91 \\
114 \\
42 \\
81 \\
75 \\
23 \\
133 \\
30 \\
87 \\
39 \\
126 \\
30 \\
136 \\
20 \\
156 \\
\end{tabular}

$\begin{array}{r}\hline \text { Stable } \\ \hline 6 \\ 26 \\ 16 \\ 7 \\ 20 \\ 35 \\ 45 \\ 10 \\ 29 \\ 26 \\ 11 \\ 44 \\ 2 \\ 28 \\ 25 \\ 50 \\ 5 \\ 48 \\ 7 \\ 55 \\ \hline\end{array}$

\begin{tabular}{r}
\hline Ineffe \\
\hline 2 \\
15 \\
13 \\
15 \\
10 \\
35 \\
34 \\
11 \\
30 \\
15 \\
5 \\
40 \\
9 \\
26 \\
10 \\
21 \\
24 \\
45 \\
0 \\
45
\end{tabular}

$\frac{\chi^{2}}{4.595} \frac{P}{0.032}$

2

15

13

15

10

35

34

11

30

15

5

40

9

26

10

14.088

0.0002

$99.860<0.0001$

$\begin{array}{ll}4.749 & 0.029\end{array}$

$\begin{array}{ll}4.749 & 0.029\end{array}$

$1.668 \quad 0.434$

$2.475 \quad 0.115$

$0.059 \quad 0.806$

Tg, thyroglobulin.

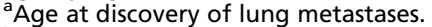

${ }^{\text {b}} \mathrm{Tg}$ level following $\mathrm{L}-\mathrm{T}_{4}$ withdrawal $(\mathrm{ng} / \mathrm{ml})$.

'Brain in one patient, parapharyneal in eight patients, liver in one patient, kidney in two patients and soft tissue in two patients. 
Table 2 Multivariate analysis of response factors of ${ }^{131}$ I therapy.

\begin{tabular}{|c|c|c|c|c|c|c|}
\hline \multirow[b]{2}{*}{ Factors } & \multicolumn{3}{|c|}{ Changes in serum $\mathbf{~ T g}$} & \multicolumn{3}{|c|}{ Imaging changes of lung metastases } \\
\hline & $\begin{array}{l}\text { Adjusted odds } \\
\text { ratios }\end{array}$ & $95 \% \mathrm{Cl}$ & $P$ value & $\begin{array}{l}\text { Adjusted odds } \\
\text { ratios }\end{array}$ & $95 \% \mathrm{Cl}$ & $P$ value \\
\hline Age $(\geq 40 /<40)$ & 1.414 & $1.040-1.934$ & 0.027 & 1.422 & $1.051-1.923$ & 0.022 \\
\hline Gender (females/males) & 0.742 & $0.446-1.233$ & 0.248 & 1.483 & $0.890-2.470$ & 0.130 \\
\hline Time of lung metastasis (initial/developed) & & & & 0.766 & $0.468-1.255$ & 0.290 \\
\hline Tg level ${ }^{a}$ & 1.264 & $0.856-1.867$ & 0.239 & 1.375 & $0.948-1.995$ & 0.093 \\
\hline $\begin{array}{l}\text { Site (lung combined with other organs } \\
\text { metastases/only lung) }\end{array}$ & 2.562 & $1.443-4.549$ & 0.001 & 1.575 & $0.890-2.785$ & 0.118 \\
\hline Node size $^{c}$ & 2.553 & $1.111-5.865$ & 0.027 & 4.464 & $1.863-10.697$ & 0.0008 \\
\hline
\end{tabular}

${ }^{a} \mathrm{Tg}$ level following $\mathrm{L}-\mathrm{T}_{4}$ withdrawal (ng/ml).

brain in one patient, parapharyneal in eight patients, liver in one patient, kidney in two patients and soft tissue in two patients.

${ }^{C}$ Node size: for serum Tg group, CT was positive/negative; for anatomical imaging group, CT was nodule $>1 / \leq 1 \mathrm{~cm}$.

on chest CT; and undetectable serum Tg or $\leq 10 \mathrm{ng} / \mathrm{ml}$ in the hypothyroid status and in the absence of $\mathrm{TgAb}$.

\section{Statistical analysis}

SPSS version 17.0 and SAS version 13.0 were used for statistical analyses. All data are expressed as mean \pm s.D., proportions or absolute numbers. All the factors that may have affected serum $\mathrm{Tg}$ and anatomical imaging in the pulmonary metastases were analyzed by univariate analysis and confirmed by the $\chi^{2}$ test. Binary logistic regression analysis was used to determine which factors contributed to the outcome of ${ }^{131} \mathrm{I}$ therapy response. Kaplan-Meier survival analysis was applied to evaluate

Table 3 Changes of anatomical imaging for 229 DTC patients with ${ }^{131} \mathrm{I}$-avid lung metastases after ${ }^{131}$ I therapy: univariate statistical analysis.

\begin{tabular}{l}
\hline Factors \\
\hline Age $^{\mathrm{a}}$ \\
$0-19$ \\
$20-39$ \\
$40-59$ \\
$\geq 60$ \\
Gender \\
Male \\
Female \\
Pathological type \\
Papillary \\
Follicular \\
Operation method \\
Lobectomy or subtotal thyroidectomy \\
Total thyroidectomy \\
Time of lung metastasis \\
Initial \\
Developed \\
Tg level \\
$1-100$ \\
$100-1000$ \\
$>1000$ \\
Metastatic site \\
Lung and lung +lymph node \\
Lung + bone and lung + other organs \\
Node size (cm) \\
$\leq 1$ \\
$>1$ \\
Total \\
\hline
\end{tabular}

\begin{tabular}{|c|c|}
\hline No. of patients & CR \\
\hline $14(6.11 \%)$ & 3 \\
\hline $99(43.23 \%)$ & 20 \\
\hline $76(33.19 \%)$ & 14 \\
\hline $40(17.47 \%)$ & 1 \\
\hline $86(37.55 \%)$ & 14 \\
\hline $143(62.45 \%)$ & 24 \\
\hline $175(76.42 \%)$ & 30 \\
\hline $54(23.58 \%)$ & 8 \\
\hline $132(57.64 \%)$ & 23 \\
\hline $97(42.36 \%)$ & 15 \\
\hline $33(14.41 \%)$ & 15 \\
\hline $196(85.59 \%)$ & 23 \\
\hline $37(16.16 \%)$ & 11 \\
\hline $121(52.84 \%)$ & 25 \\
\hline $71(31.00 \%)$ & 2 \\
\hline $175(76.42 \%)$ & 28 \\
\hline $54(23.58 \%)$ & 10 \\
\hline $212(92.58 \%)$ & 36 \\
\hline $17(7.42 \%)$ & 2 \\
\hline 229 & 38 \\
\hline
\end{tabular}

$\begin{array}{r}\hline \text { PR } \\ \hline 4 \\ 43 \\ 36 \\ 17 \\ 49 \\ 51 \\ 76 \\ 24 \\ 57 \\ 43 \\ 11 \\ 89 \\ 13 \\ 51 \\ 36 \\ 93 \\ 7 \\ 96 \\ 4 \\ 100 \\ \hline\end{array}$

$\begin{array}{r}\hline \text { SD } \\ \hline 7 \\ 31 \\ 24 \\ 14 \\ 20 \\ 56 \\ 59 \\ 17 \\ 43 \\ 33 \\ \\ 5 \\ 71 \\ 9 \\ 36 \\ 31 \\ 43 \\ 33 \\ 74 \\ 76 \\ \hline\end{array}$

\begin{tabular}{|c|c|c|}
\hline PD & $\chi^{2}$ & $\boldsymbol{P}$ \\
\hline $\begin{array}{l}0 \\
5 \\
2 \\
8\end{array}$ & 6.109 & 0.013 \\
\hline $\begin{array}{r}3 \\
12\end{array}$ & 5.058 & 0.024 \\
\hline $\begin{array}{r}10 \\
5\end{array}$ & 0.993 & 0.802 \\
\hline $\begin{array}{l}9 \\
6\end{array}$ & & \\
\hline $\begin{array}{r}2 \\
13\end{array}$ & 13.100 & 0.0003 \\
\hline $\begin{array}{l}4 \\
9 \\
2\end{array}$ & 4.114 & 0.042 \\
\hline $\begin{array}{r}11 \\
4\end{array}$ & 8.044 & 0.004 \\
\hline $\begin{array}{r}6 \\
9 \\
15\end{array}$ & 15.801 & $<0.0001$ \\
\hline
\end{tabular}

CR, complete response; PR, partial response; SD, stable disease; PD, progressive disease.

${ }^{a}$ Age at discovery of lung metastases.

${ }^{\mathrm{b}} \mathrm{Tg}$ level following $\mathrm{L}-\mathrm{T}_{4}$ withdrawal $(\mathrm{ng} / \mathrm{ml})$.

'Brain in one patient, parapharyneal in eight patients, liver in one patient, kidney in two patients and soft tissue in two patients. 
Table 4 Comparison by univariate statistical analysis between the groups of DTC patients with ${ }^{131} \mathrm{I}$-avid and non- ${ }^{131} \mathrm{I}$-avid lung metastases.

\begin{tabular}{l} 
Factors \\
\hline Age $^{\mathrm{a}}$ \\
$0-19$ \\
$20-39$ \\
$40-59$ \\
$\geq 60$ \\
Gender \\
Male \\
Female \\
Pathological type \\
Papillary \\
Foliicular \\
Operation method \\
Lobectomy or subtotal thyroidectomy \\
Total thyroidectomy \\
Time of lung metastasis \\
Initial \\
Developed \\
Metastatic site \\
Lung and lung +lymph node \\
Lung + bone and lung +other organs ${ }^{\mathrm{b}}$ \\
Node size (cm) \\
CT-negative \\
$\leq 1$ \\
$>1$ \\
Total
\end{tabular}

\begin{tabular}{c}
\hline No. of patients \\
\hline $19(5.11 \%)$ \\
$129(34.68 \%)$ \\
$141(37.90 \%)$ \\
$83(22.31 \%)$ \\
$134(36.02 \%)$ \\
$238(63.98 \%)$ \\
$288(77.42 \%)$ \\
$84(22.58 \%)$ \\
$207(55.65 \%)$ \\
$165(44.35 \%)$ \\
$43(11.56 \%)$ \\
$329(88.44 \%)$ \\
$298(80.11 \%)$ \\
$74(19.89 \%)$ \\
$28(7.53 \%)$ \\
$313(84.14 \%)$ \\
$31(8.33 \%)$ \\
372
\end{tabular}

$\begin{array}{r}\hline{ }^{131} \mathbf{I} \text {-avid } \\ \hline 18 \\ 112 \\ 86 \\ 40 \\ 95 \\ 161 \\ 193 \\ 63 \\ 140 \\ 116 \\ 39 \\ 217 \\ 197 \\ 59 \\ 27 \\ 212 \\ 17 \\ 256 \\ \hline\end{array}$

\begin{tabular}{c}
\hline Non- ${ }^{\mathbf{1 3 1}}$ I-avid \\
\hline 1 \\
17 \\
55 \\
43 \\
39 \\
77 \\
95 \\
21 \\
67 \\
49 \\
4 \\
112 \\
101 \\
15 \\
1 \\
101 \\
14 \\
116 \\
\hline
\end{tabular}

$\frac{\chi^{2}}{43.639}$

\begin{tabular}{c}
\hline $\boldsymbol{P}$ \\
\hline$<0.0001$
\end{tabular}

${ }^{\mathrm{a}}$ Age at discovery of lung metastases.

${ }^{b}$ For ${ }^{131}$ l-avid group: brain in one patient, parapharyneal in eight patients, liver in one patient, kidney in two patients and soft tissue in two patients; for non- ${ }^{131}$ I-avid group: brain in two patient, parapharyneal in two patients, liver in one patient, kidney in one patient and soft tissue in one patient.

the effect of different variables on survival and the differences between the groups were compared using the log-rank test. Multivariate analysis was performed to assess the relationship between survival time and several variables simultaneously. For analyses of this type, Cox proportional hazards model was used. A $P$ value $<0.05$ was considered to indicate statistical significance.

\section{Results}

\section{Characteristics of patients with pulmonary metastases}

There were 372 enrolled patients with a predominance of females $($ male $=134$, female $=238$ and male:female ratio $=1: 1.78)$. The ratio was lower than that observed in the general series of DTC patients, which means that pulmonary metastases are relatively more frequent in male patients. The mean age at diagnosis was $54.25 \pm$ 16.08 years (range, 12-86 years) and the mean follow-up period was $59.48 \pm 40.41$ months. The initial pulmonary metastases were detected in 43 patients and the remaining 329 patients were diagnosed at follow-up (developed pulmonary metastases). Chest CT showed that pulmonary metastases were negative in $28(28 / 372$, $7.53 \%)$ patients, micronodular $(\leq 1 \mathrm{~cm})$ in $313(313 / 372$, $84.14 \%)$ patients and macronodular $(>1 \mathrm{~cm})$ in 31 $(31 / 372,8.33 \%)$ patients. ${ }^{131}$ I-avid pulmonary metastases

Table 5 Multivariate analysis between the groups of DTC patients with ${ }^{131} \mathrm{I}$-avid and non- ${ }^{131} \mathrm{I}$-avid lung metastases.

\section{Factors}

Age $(\geq 40 /<40)$

Time of lung metastasis (initial/developed)

Site (lung combined with other organs metastases $\%$ only lung)

Nodule size $(>1 / \leq 1 \mathrm{~cm})$

\begin{tabular}{c} 
Adjusted odds ratios \\
\hline 2.494 \\
1.028 \\
2.203 \\
6.335 \\
\hline
\end{tabular}

\begin{tabular}{c}
$\mathbf{9 5 \%} \mathbf{C l}$ \\
\hline $1.865-3.350$ \\
$0.644-1.641$ \\
$1.260-3.850$ \\
$3.269-12.278$ \\
\hline
\end{tabular}

\begin{tabular}{c} 
P value \\
\hline$<0.0001$ \\
0.909 \\
0.005 \\
$<0.0001$
\end{tabular}

${ }^{a}$ For ${ }^{131}$ I-avid group: brain in one patient, parapharyneal in eight patients, liver in one patient, kidney in two patients and soft tissue in two patients; for non- ${ }^{131}$ I-avid group: brain in two patient, parapharyneal in two patients, liver in one patient, kidney in one patient and soft tissue in one patient. 
was observed in $256(256 / 372,68.82 \%)$ patients and non- ${ }^{131}$ I-avid pulmonary metastases was found in 116 (116/372, 31.18\%) patients. The median cumulative radioiodine dose was $18.315 \mathrm{GBq}(495 \mathrm{mCi})$ with a range of $3.7-86.95 \mathrm{GBq}(100-2350 \mathrm{mCi})$. A single dose was given to 37 patients, two doses to 61 patients and greater than or equal to three doses to the remaining 274 patients.

\section{Changes in serum $\mathrm{Tg}$ level after ${ }^{131} \mathrm{I}$ therapy}

Changes in serum Tg in 256 DTC patients with ${ }^{131} \mathrm{I}$-avid pulmonary metastases after radioiodine therapy are shown in Table 1. ${ }^{131}$ I therapy was effective in 156 patients (BR, effective rate, 60.9\%) and the average serum $\mathrm{Tg}$ varied from 683 (range, 17-6957) to 311 (range, 0-1407) $\mathrm{ng} / \mathrm{ml}$. Stabilization of $\mathrm{Tg}$ was seen in 55 patients (stable rate, $21.5 \%$ ) after ${ }^{131}$ I therapy and the average serum Tg varied from 791 (range, 27-8027) to 692 (range, 15-8395) $\mathrm{ng} / \mathrm{ml}$. Treatment was ineffective in 45 patients (ineffective rate, $17.6 \%$ ) and the average serum $\mathrm{Tg}$ increased from 847 (range, 19-15 955) to 1301 (range, 26-20 475) $\mathrm{ng} / \mathrm{ml}$.

Univariate analysis showed that changes in serum Tg were influenced by age, sex, the initial Tg level, combination with other distant metastases and pulmonary node size ( $P$ valves $0.0321,0.0210,<0.0001$, 0.0002 and 0.0293 respectively). Multivariate analyses revealed that age, combination with other distant metastases and pulmonary node size had a significant impacts on $\mathrm{Tg}$ changes $(P$ values $0.0270,0.0013$ and 0.0272 respectively; shown in Table 2). Significantly decreased serum $\mathrm{Tg}$ (BR) and favorable efficacy were observed in patients who were aged $<40$ years, only had pulmonary metastases and 'fine miliaric' nodes (negative chest CT).

\section{Changes in chest CT after ${ }^{131}$ I therapy}

Of the 229 pulmonary metastases patients with positive chest CT findings, only 38 achieved CR (16.6\%), 100 PR (43.7\%), 76 SD (33.2\%) and 15 had PD (6.5\%). Univariate analyses of the factors influencing the changes of CT are shown in Table 3. Age, sex, time of lung metastases, initial Tg level, combination with other distant metastases and pulmonary node size were significantly associated with CT changes ( $P$ values $0.0134,0.0245,0.0003$, $0.0425,0.0046$ and $<0.0001$ respectively). Multivariate analyses revealed that age $<40$ years and node size $<1 \mathrm{~cm}$ had favorable effect on CT changes ( $P$ values 0.0223 and 0.0008; Table 2).

Table 6 Survival for patients with lung metastatic differentiated thyroid cancer.

\begin{tabular}{|c|c|c|c|c|c|c|c|}
\hline Factor & $\begin{array}{l}\text { No. of } \\
\text { patients }\end{array}$ & $\begin{array}{l}\text { 5-year } \\
\text { survival } \\
\text { rate }(\%)\end{array}$ & $\begin{array}{l}10 \text {-year } \\
\text { survival } \\
\text { rate }(\%)\end{array}$ & $\begin{array}{l}\text { 15-year } \\
\text { survival } \\
\text { rate }(\%)\end{array}$ & $\begin{array}{l}\text { Univariate } \\
P \text { value }\end{array}$ & $\begin{array}{l}\text { Multivariate } \\
\text { P value }\end{array}$ & $\begin{array}{c}\text { Hazard ratio } \\
(95 \% \mathrm{CI})\end{array}$ \\
\hline Age (years) & & & & & $<0.0001$ & 0.00015 & \\
\hline$<40$ & $130(50.78 \%)$ & 90.7 & 79.5 & 66.9 & & & 1 \\
\hline$\geq 40$ & $126(49.22 \%)$ & 77.6 & 59.2 & 32.7 & & & $1.7(1.2-2.6)$ \\
\hline Gender & & & & & NS & & \\
\hline Males & $95(37.11 \%)$ & 81.7 & 67.6 & 55.8 & & & \\
\hline Females & $161(62.89 \%)$ & 85.3 & 72.2 & 62.9 & & & \\
\hline Pathological type & & & & & NS & & \\
\hline Papillary & $193(75.39 \%)$ & 88.0 & 73.6 & 60.4 & & & \\
\hline Follicular & $63(24.61 \%)$ & 80.5 & 69.2 & 58.8 & & & \\
\hline Time of lung metastasis & & & & & NS & & \\
\hline Initial & $39(15.23 \%)$ & 86.9 & 73.3 & 64.8 & & & \\
\hline Developed & $217(84.77 \%)$ & 89.5 & 75.4 & 62.3 & & & \\
\hline Metastatic site & & & & & $<0.0001$ & $<0.0001$ & \\
\hline Lung and lung + lymph node & $197(76.95 \%)$ & 90.0 & 73.6 & 60.9 & & & 1 \\
\hline $\begin{array}{l}\text { Lung }+ \text { bone and lung }+ \\
\text { other organs }\end{array}$ & $59(23.05 \%)$ & 45.9 & 34.3 & 17.2 & & & $2.3(1.7-3.6)$ \\
\hline Node size $(\mathrm{cm})$ & & & & & $<0.0001$ & $<0.0001$ & \\
\hline Negative & $27(10.55 \%)$ & 100.0 & 90.9 & 75.8 & & & 1 \\
\hline$\leq 1$ & $212(82.81 \%)$ & 88.5 & 68.9 & 50.1 & & & $1.9(1.1-3.4)$ \\
\hline$>1$ & $17(6.64 \%)$ & 59.2 & 30.6 & 0 & & & $3.5(2.1-5.8)$ \\
\hline${ }^{131} \mid$ & & & & & $<0.0001$ & $<0.0001$ & \\
\hline Yes & $256(68.82 \%)$ & 87.0 & 69.2 & 56.2 & & & 1 \\
\hline No & $116(31.18)$ & 69.7 & 38.1 & 20.9 & & & $2.1(1.3-3.2)$ \\
\hline
\end{tabular}




\section{Patients who achieved complete cure}

Sixty-two patients achieved complete cure after radioiodine therapy for pulmonary metastases. Mean age was 30 years old (range, 12-56 years). Fifty-eight patients had pulmonary metastases only and the remaining four patients had single bone metastases, which were treated with surgery and external radiotherapy. Serum Tg level measured after withdrawal of $\mathrm{L}_{-} \mathrm{T}_{4}$ treatment were undetectable in 39 patients and $<10 \mathrm{ng} / \mathrm{ml}$ in the remaining 23 patients. Complete cure occurred in 24 patients with a negative chest CT examination at discovery of pulmonary metastases, 36 with micronodular metastases and two with macronodular metastases. The mean cumulative dose of ${ }^{131} \mathrm{I}$ administered to the 62 patients was $11.248 \mathrm{GBq}(304 \mathrm{mCi})$, six with $<7.4 \mathrm{GBq}$ (200 mCi), 40 with 7.4-14.8 GBq (200-400 mCi), 13 with 14.8-22.2 GBq (400-600 $\mathrm{mCi}$ ) and only three with a cumulative activity $>22.2 \mathrm{GBq}(600 \mathrm{mCi})$. Three of these 62 patients have relapsed during follow-up period.

\section{Patients with no ${ }^{131}$ I uptake}

A total of 116 patients had non-functioning pulmonary metastases. Fifteen of these patients had multiple organ involvement. The non-functioning lung metastases occurred late (found 6 months or more after thyroidectomy) in 112 patients. The proportion of patients older than 40 years was much higher (non- ${ }^{131}$ I-avid vs ${ }^{131} \mathrm{I}$-avid $=84.5 \%$ vs $49.2 \%$ ).

After univariate analysis between the patients with ${ }^{131} \mathrm{I}$-avid and non- ${ }^{131} \mathrm{I}$-avid pulmonary metastases (Table 4), the significant factors related to the ability of ${ }^{131}$ I uptake were age, pathological type, time of discovery lung metastases, combination with other distant metastases and node size. After multivariate analysis (Table 5), only age, combination with other distant metastases and node size remained significant for ${ }^{131}$ I uptake.

\section{Survival rate and prognostic survival factors}

The survival rate and prognostic survival factors are listed in Table 6. Survival rate in ${ }^{131} \mathrm{I}$-avid patients was $87.0 \%$ at 5 years, $69.2 \%$ at 10 years and $56.2 \%$ at 15 years. In non- ${ }^{131}$ I-avid patients, survival rate was $69.7 \%$ at 5 years, $38.1 \%$ at 10 years and $20.9 \%$ at 15 years (Fig. 1). For

${ }^{131}$ I-avid patients, the univariate analysis showed that survival rate was affected by age, combination with other distant metastases and pulmonary node size $(P$ value $<0.0001$ for all the factors). Prognostic variables were strongly inter-related. After adjusting for other significant factors, Cox's regression analyses showed that the three variables remained significant for survival ( $P$ valves $0.00015,<0.0001$ and $<0.0001$ respectively).

The survival rate at 10 years was $79.5 \%$ in patients aged $<40$ years and $59.2 \%$ in patients older than 40 years (Fig. 2). For patients with only lung metastases, the survival rate at 10 years was $73.6 \%$, which was significantly higher than patients with multiple organ metastases (for whom the 10 -year survival rate was $34.3 \%$; Fig. 3). The 10 -year survival rate of $90.9 \%$ in patients with negative chest CT was higher than the $68.9 \%$ survival rate observed in patients with micronodular metastases $(\leq 1 \mathrm{~cm})$ and the $30.6 \%$ observed in those with macronodular metastases ( $>1 \mathrm{~cm}$ ) (Fig. 4).

\section{Discussion}

We studied a large number of DTC patients with pulmonary metastases. We assessed the effect of radioiodine therapy and investigated the prognostic factors for survival for DTC patients with pulmonary metastases. A few studies have looked primarily at the clinical management and outcome of patients with DTC presenting with lung metastases $(1,2$, $3,4,8,11,12,14,15)$. Our study differed from earlier investigations in three regards: i) we included a larger patient sample; ii) when evaluating the effect of radioiodine therapy, we explored only the patients with ${ }^{131}$ I-avid pulmonary metastases and the factors that affected

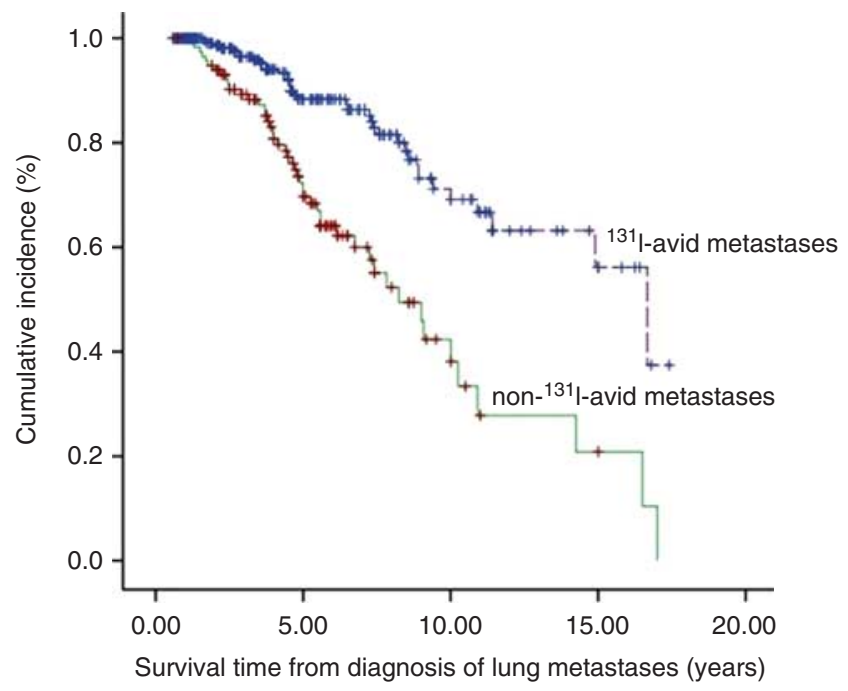

Figure 1

Overall survival for patients with lung metastases by ${ }^{131} \mathrm{I}$-avid and non- ${ }^{131}$ I-avid. 


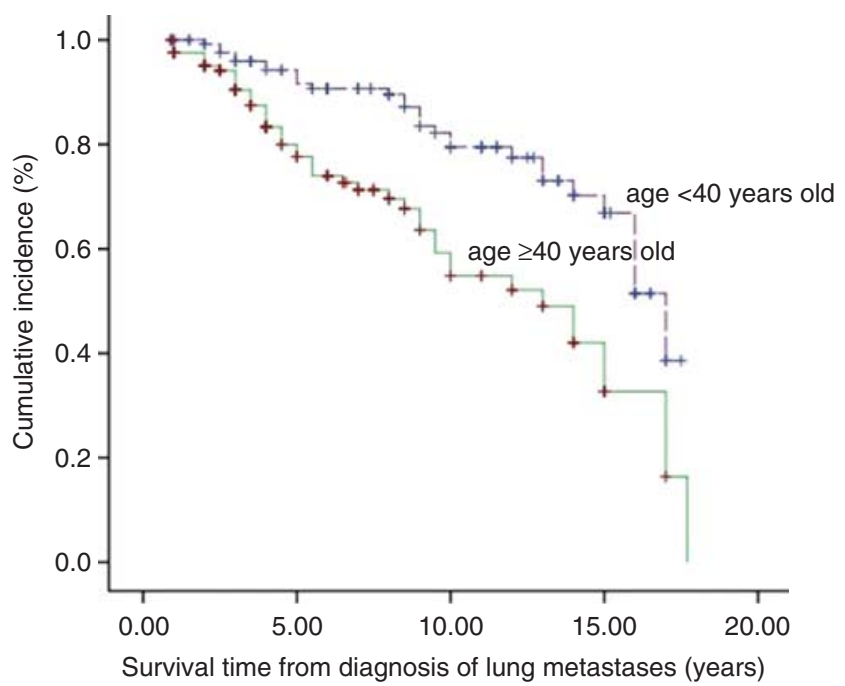

Figure 2

Overall survival for patients with lung metastases by age at diagnosis.

${ }^{131}$ I uptake were discussed for non- ${ }^{131}$ I-avid lung metastases and iii) the parameters of Tg level and chest CT were selected as an evaluation index and discussed.

For patients with non- ${ }^{131}$ I-avid (radioiodine-refractory) pulmonary disease, the benefit of radioiodine therapy was very limited. It was not appropriate for evaluation of the efficacy of ${ }^{131}$ I therapy in these patients. Loss of radioiodine avidity is associated with a poor outcome. Several studies have reported that DTC patients with ${ }^{131}$ I-avidity have longer survival rates than those with non- ${ }^{131}$ I-avid pulmonary metastasis $(15,16,17,18)$. In agreement with the previous studies, our patients with non- ${ }^{131}$ I-avid disease had a 10 -year OS rate of $38.1 \%$ compared to $69.2 \%$ for ${ }^{131} \mathrm{I}$-avid metastases $(P<0.0001)$. We further investigated the factors related to ${ }^{131}$ I uptake. After univariate and multivariate analyses, age $>40$ years $(P<0.0001)$, combination with other distant metastases $(P=0.0056)$ and macronodular lesions $(P<0.0001)$ were independently significant predictors for non- ${ }^{131} \mathrm{I}$-avid pulmonary metastases.

Numerous studies have been performed on the diagnostic value of serum $\mathrm{Tg}$ measurement and have found that it is a highly sensitive means of detecting metastases (19). Moreover, Tg level was an independent prognostic indicator for disease-free remission and death $(20,21)$. It was thought that the serum Tg level under TSH elevation $(>30 \mathrm{mU} / \mathrm{l})$ was the most reliable indicator for persistent or recurrent disease. Therefore, the change in serum Tg levels (after the withdrawal of L-T4 over the course of 3-4 weeks) was considered to be a prognostic indicator to assess the efficacy of ${ }^{131}$ I therapy for pulmonary metastases. We found a significant decrease in serum $\mathrm{Tg}$ (BR) in 156 patients (60.9\% effective rate). Multivariate analyses revealed that age $\leq 40$ years, only lung metastases and 'fine miliaric' nodes (negative chest CT) were the favorable factors. These corresponded well with the favorable prognostic factors of OS and suggested that decreased serum Tg indicates the effectiveness of radioiodine therapy. However, serum Tg was only reliable if $\mathrm{TgAb}$ was undetectable, otherwise serum Tg level could have been falsely lowered (21). Thus, patients with positive antibodies were excluded from the study. The size of pulmonary metastases was usually $<1 \mathrm{~cm}$, which was below the spatial resolution of chest X-ray. Chest CT could enhance the diagnostic accuracy in most patients with normal chest X-ray and had an additive effect to ${ }^{131}$ I-WBS and Tg level (11). According to nodule size on chest CT, pulmonary metastases were divided into three categories: i) normal $(n=27)$; ii) micronodular $(\leq 1 \mathrm{~cm}$, $n=212$ ) and iii) macronodular $(>1 \mathrm{~cm}, n=17)$. The significance of this classification was that it was related to radioiodine efficacy. Micronodular pattern of lung metastases was invariably related to good ${ }^{131}$ I uptake, while macronodular metastases frequently showed poor ${ }^{131} \mathrm{I}$ uptake (14). In our study, multivariate analyses revealed that patients with node size $<1 \mathrm{~cm}$ had a favorable

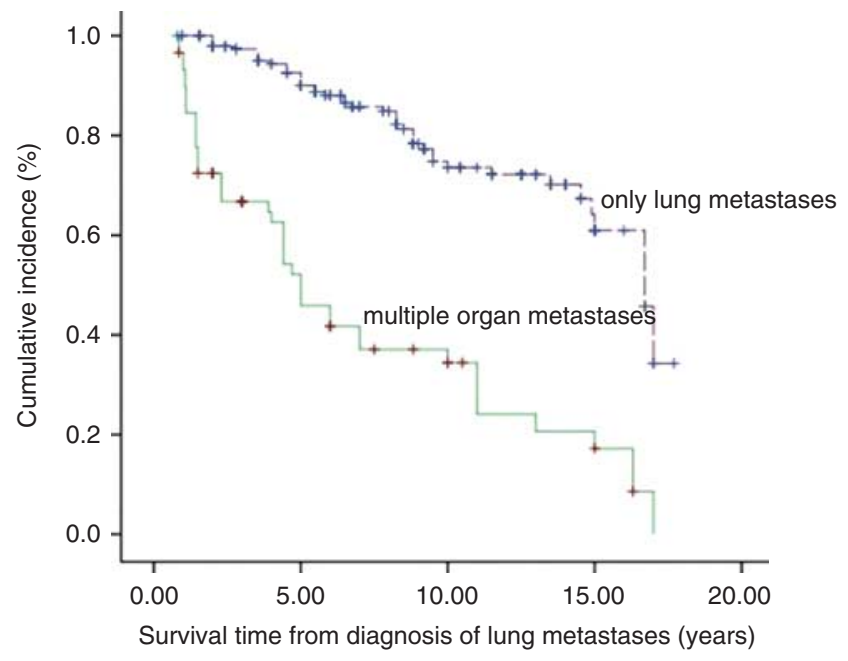

Figure 3

Overall survival for patients with lung metastases by sites of metastasis. 


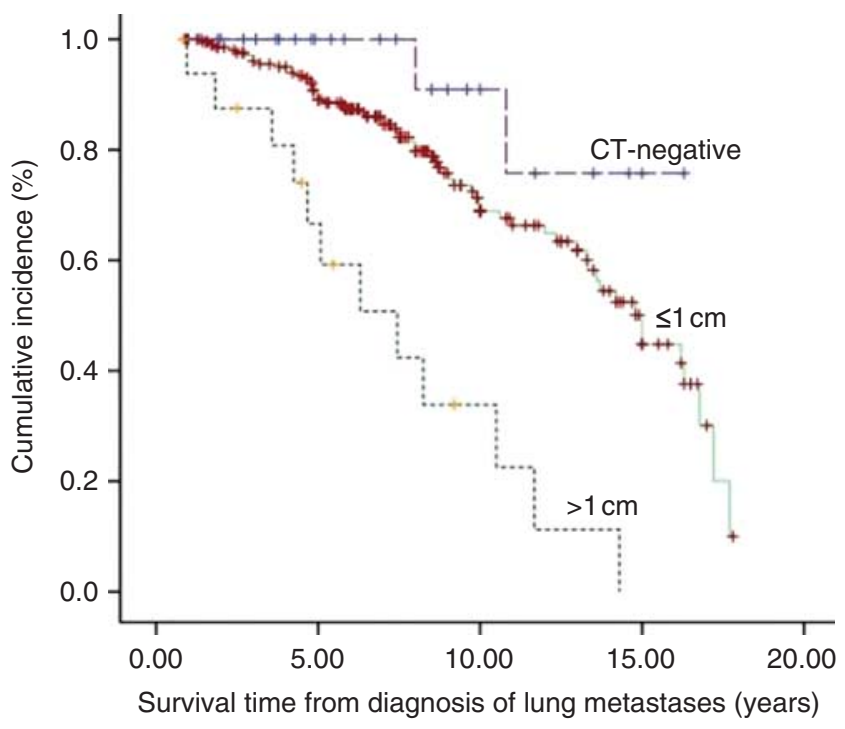

\section{Figure 4}

Overall survival for patients with lung metastases by pulmonary node size.

response on changes in $\mathrm{CT}$ imaging $(P=0.0008, \mathrm{OR}=$ 4.464, 95\% CI, 1.863-10.697). Complete remission following ${ }^{131}$ I therapy always occurred in patients with micronodular metastases, especially for 'fine miliaric' nodes. The 10-year survival rate was significantly higher in category 1 (90.9\%) patients, lower in category 2 (68.9\%) and only $30.6 \%$ in category 3 patients.

In our cohort, for patients with ${ }^{131} \mathrm{I}$-avid pulmonary metastases, the 5-, 10- and 15-year OS rates were 87.0, 69.2 and $56.2 \%$ respectively. This corresponded with the result of those reported in the previous studies $(8,15,22,23,24)$. Cox's regression analysis showed that the three variables, including age, combination with other distant metastases and pulmonary node size, were significant predictor for survival ( $P$ valves $0.00015,<0.0001$ and $<0.0001$ respectively). Age is known to be an independent prognostic factor for mortality in DTC $(14,25,26)$, and the mortality risk increases with age. In our series, survival rate at 10 years was $79.5 \%$ in the patients younger than 40 years of age, while it was only $59.2 \%$ in the patients older than 40 years. Although pulmonary metastases with ${ }^{131}$ I uptake may be stable for several years or decades, all metastases should be treated at an early stage. Favorable responses have been observed in patients with small metastases. Previously, Casara et al. (14) showed that micronodular metastasis susceptible to ${ }^{131}$ I therapy had a good prognosis, whereas macronodular metastasis of non- ${ }^{131} \mathrm{I}$ - avid lesions had a poor prognosis. As in our study, patients with normal chest CT can achieve a CR with radioiodine alone for long periods of time. Macronodular metastases frequently show poor ${ }^{131}$ I uptake $(14 / 31,45.2 \%)$ and poor survival rate (10-year, $\mathrm{OS}=30.6 \%)$.

\section{Limitations}

First, our study was retrospective. During the long study period, a few clinical data were missing, which might have caused bias in the data selection. Second, in the diagnosis of lung metastasis in non- ${ }^{131} \mathrm{I}$-avid patients, the histopathological confirmation was not always possible, particularly in patients with small ('fine miliaric' or micronodular) lesions. Therefore, they were clinically diagnosed based on their CT images and elevated Tg levels. However, we cannot completely rule out the possibility of other conditions, such as incidental primary lung cancers or benign lung diseases such as inflammation.

\section{Summary}

In conclusion, our study indicated that most ${ }^{131} \mathrm{I}$-avid pulmonary metastases from DTC can obtain partial or complete remission after ${ }^{131} \mathrm{I}$ therapy. Younger patients ( $<40$ years old) with only pulmonary metastases and small ('fine miliaric' or micronodular) metastases appear to have relative favorable outcomes. In contrast, patients with non ${ }^{131}$ I-avid pulmonary metastases who do not respond to radioiodine treatment have a worse prognosis, and ${ }^{131} \mathrm{I}$ treatment should be abandoned and other treatment modalities used when tumour progression has been documented.

Declaration of interest

The authors declare that there is no conflict of interest that could be perceived as prejudicing the impartiality of the research reported.

Funding

This study was sponsored by the National Natural Science Foundation of China (No. 81271611 and 81201115 ).

Author contribution statement

H-J Song, Z-L Qiu and Q-Y Luo conceived and designed the paper. H-J Song, C-T Shen and W-J Wei collected the data. H-J Song and Z-L Qiu analyzed the data. H-J Song wrote the paper.

\section{References}

1 Massin JP, Savoie JC, Garnier H, Guiraudon G, Leger FA \& Bacourt F. Pulmonary metastases in differentiated thyroid carcinoma. Study of 58 
cases with implications for the primary tumor treatment. Cancer 1984 53 982-992. (doi:10.1002/1097-0142(19840215)53:4<982::AIDCNCR2820530427>3.0.CO;2-E)

2 Ruegemer JJ, Hay ID, Bergstralh EJ, Ryan JJ, Offord KP \& Gorman CA. Distant metastases in differentiated thyroid carcinoma: a multivariate analysis of prognostic variables. Journal of Clinical Endocrinology and Metabolism 198867 501-508. (doi:10.1210/jcem-67-3-501)

3 Casara D, Rubello D, Saladini G, Gallo V, Masarotto G \& Busnardo B. Distant metastases in differentiated thyroid cancer: long-term results of radioiodine treatment and statistical analysis of prognostic factors in 214 patients. Tumori 199177 432-436.

4 Samaan NA, Schultz PN, Haynie TP \& Ordonez NG. Pulmonary metastasis of differentiated thyroid carcinoma: treatment results in 101 patients. Journal of Clinical Endocrinology and Metabolism 198560 376-380. (doi:10.1210/jcem-60-2-376)

5 Jonklaas J, Sarlis NJ, Litofsky D, Ain KB, Bigos ST, Brierley JD, Cooper DS, Haugen BR, Ladenson PW, Magner J et al. Outcomes of patients with differentiated thyroid carcinoma following initial therapy. Thyroid 200616 1229-1242. (doi:10.1089/thy. 2006.16.1229)

6 Lang BH, Wong KP, Cheung CY, Wan KY \& Lo CY. Evaluating the prognostic factors associated with cancer specific survival of differentiated thyroid carcinoma presenting with distant metastasis. Annals of Surgical Oncology 201320 1329-1335. (doi:10.1245/s10434-012-2711-x)

7 Mazzaferri EL \& Kloos RT. Current approaches to primary therapy for papillary and follicular thyroid cancer. Journal of Clinical Endocrinology and Metabolism 2001 86 1447-1463. (doi:10.1210/ jcem.86.4.7407)

8 Durante C, Haddy N, Baudin E, Leboulleux S, Hartl D, Travagli JP, Caillou B, Ricard M, Lumbroso JD, De Vathaire F et al. Long-term outcome of 444 patients with distant metastases from papillary and follicular thyroid carcinoma: benefits and limits of radioiodine therapy. Journal of Clinical Endocrinology and Metabolism 200691 2892-2899. (doi:10.1210/jc.2005-2838)

9 Watkinson JC. The British Thyroid Association guidelines for the management of thyroid cancer in adults. Nuclear Medicine Communications 200425 897-900. (doi:10.1097/00006231200409000-00006)

10 Cooper DS, Doherty GM, Haugen BR, Kloos RT, Lee SL, Mandel SJ, Mazzaferri EL, McIver B, Pacini F, Schlumberger M et al. American Thyroid Association (ATA) Guidelines Taskforce on Thyroid Nodules and Differentiated Thyroid Cancer. Revised American Thyroid Association management guidelines for patients with thyroid nodules and differentiated thyroid cancer. Thyroid 200919 1167-1214. (doi:10.1089/thy.2009.0110)

11 Ilgan S, Karacalioglu AO, Pabuscu Y, Atac GK, Arslan N, Ozturk E, Gunalp B \& Ozguven MA. Iodine-131 treatment and high-resolution CT: results in patients with lung metastases from differentiated thyroid carcinoma. European Journal of Nuclear Medicine and Molecular Imaging 200431 825-830. (doi:10.1007/s00259004-1460-x)

12 Samuel AM, Rajashekharrao B \& Shah DH. Pulmonary metastases in children and adolescent with well-differentiated thyroid cancer. Journal of Nuclear Medicine 199839 1531-1536.

13 Qiu ZL, Song HJ, Xu YH \& Luo QY. Efficacy and survival analysis of ${ }^{131}$ I therapy for bone metastases form differentiated thyroid cancer. Journal of Clinical Endocrinology and Metabolism 201196 3078-3086. (doi:10.1210/jc.2011-0093)
14 Casara D, Rubello D, Saladini G, Masarotto G, Favero A, Girelli ME \& Busnardo B. Different features of pulmonary metastases in differentiated thyroid cancer: natural history and multivariate statistical analysis of prognostic variables. Journal of Nuclear Medicine 199334 1626-1631.

15 Cho SW, Choi HS, Yeom GJ, Lim JA, Moon JH, Park do J, Chung JK, Cho BY, Yi KH \& Park YJ. Long-term prognosis of differentiated thyroid cancer with lung metastasis in Korea and its prognostic factors. Thyroid 201424 277-286. (doi:10.1089/thy.2012.0654)

16 Pelizzo MR, Boschin IM, Toniato A, Piotto A, Pagetta C, Gross MD, Al-Nahhas A \& Rubello D. Papillary thyroid carcinoma: 35 -year outcome and prognostic factors in 1858 patients. Clinical Nuclear Medicine 200732 440-444. (doi:10.1097/RLU. Ob013e31805375ca)

17 Sisson JC, Giordano TJ, Jamadar DA, Kazerooni EA, Shapiro B, Gross MD, Zempel SA \& Spaulding SA. 131-I treatment of micronodular pulmonary metastases from papillary thyroid carcinoma. Cancer 199678 2184-2192. (doi:10.1002/(SICI)1097-0142 (19961115)78:10<2184::AID-CNCR21>3.0.CO;2-U)

18 Dinneen SF, Valimaki MJ, Bergstralh EJ, Goellner JR, Gorman CA \& Hay ID. Distant metastases in papillary thyroid carcinoma: 100 cases observed at one institution during 5 decades. Journal of Clinical Endocrinology and Metabolism 199580 2041-2045.

19 Lind P \& Kohlfürst S. Respective roles of thyroglobulin, radioiodine imaging, and positron emission tomography in the assessment of thyroid cancer. Seminars in Nuclear Medicine 200636 194-205. (doi:10.1053/j.semnuclmed.2006.03.002)

20 Heemstra KA, Liu YY, Stokkel M, Kievit J, Corssmit E, Pereira AM, Romijn JA \& Smit JW. Serum thyroglobulin concentrations predict disease-free remission and death in differentiated thyroid carcinoma. Clinical Endocrinology 200766 58-64.

21 Brassard M, Borget I, Edet-Sanson A, Giraudet AL, Mundler O, Toubeau M, Bonichon F, Borson-Chazot F, Leenhardt L, Schvartz C et al. Long-term follow-up of patients with papillary and follicular thyroid cancer: a prospective study on 715 patients. Journal of Clinical Endocrinology and Metabolism 201196 1352-1359. (doi:10.1210/ jc.2010-2708)

22 Mihailovic J, Stefanovic L, Malesevic M \& Markoski B. The importance of age over radioiodine avidity as a prognostic factor in differentiated thyroid carcinoma with distant metastases. Thyroid 200919 227-232. (doi:10.1089/thy.2008.0186)

23 Huang IC, Chou FF, Liu RT, Tung SC, Chen JF, Kuo MC, Hsieh CJ \& Wang PW. Long-term outcomes of distant metastasis from differentiated thyroid carcinoma. Clinical Endocrinology 201276 439-447. (doi:10.1111/j.1365-2265.2011.04231.x)

24 Sampson E, Brierley JD, Le LW, Rotstein L \& Tsang RW. Clinical management and outcome of papillary and follicular (differentiated) thyroid cancer presenting with distant metastasis at diagnosis. Cancer 2007110 1451-1456. (doi:10.1002/cncr.22956)

25 Ronga G, Filesi M, Montesano T, Di Nicola AD, Pace C, Travascio L, Ventroni G, Antonaci A \& Vestri AR. Lung metastases from differentiated thyroid carcinoma. A 40 years' experience. Quarterly Journal of Nuclear Medicine and Molecular Imaging 200448 12-19.

26 Brierley J, Tsang R, Panzarella T \& Bana N. Prognostic factors and the effect of treatment with radioactive iodine and external beam radiation on patients with differentiated thyroid cancer seen at a single institution over 40 years. Clinical Endocrinology 200563 418-427. (doi:10.1111/j.1365-2265.2005.02358.x)

Received 17 March 2015

Revised version received 21 June 2015

Accepted 23 June 2015 